\title{
USO DEL ELISA DE CAPTURA DE ANTÍGENO EN EL MONITOREO DE PORCINOS INFECTADOS NATURALMENTE CON Cysticercus cellulosae Y TRATADOS CON OXFENDAZOL
}

\author{
Yeny Tinoco F. ${ }^{1}$, Armando González Z. ${ }^{2}$, Teresa López U. ${ }^{3}$ y María Silva I. ${ }^{2}$
}

\section{Abstract}

\begin{abstract}
Taenia solium cysticercosis is a serious public health problem in developing countries around the world. At the present time, treatment with anthelmintic drugs is possible, but once applied the treatment the monitoring of the animal is limited by the high cost of the radiological techniques. Moreover, the available immunodiagnostic assays are not useful for monitoring because they detect the presence of antibodies against C. cellulosae, which remain in circulation after cysts are dead. The objective of the present study was to demonstrate the usefulness of a monoclonal antibody-based capture ELISA (AgELISA) in the monitoring of the persistence of circulating parasite antigens in animals treated against cysticercosis. For this purpose, serum samples corresponding to the monitoring of pre and post treatment of 6 pigs, naturally infected with cysticercosis and treated with oxfendazol, were evaluated. Necropsy data and evagination records were also used. It was found that the time in which the antigen titles were lower to the cut off OD values occurred on day 59 post treatment and that the mean survival time was 71.5 (59.3-83.8) days. These results seem to demonstrate that the Ag-ELISA would allow the quantitative monitoring of the behavior and persistence of circulating antigens in naturally infected animals that are treated with an antiparasitic drug. However, the monitoring of additional treated pigs are required to obtain conclusive results.
\end{abstract}

Key words: antigen capture ELISA, Ag-ELISA, cysticercosis, post treatment monitoring, parasite antigens

\section{Resumen}

La cisticercosis por Taenia soliumes una enfermedad zoonótica común en países en desarrollo y un grave problema de salud pública a nivel mundial. En la actualidad es posible tratarla mediante el uso de cisticidas, pero una vez aplicado el tratamiento, el monitoreo de la evolución del animal es limitado por el alto costo de las técnicas de radioimagen. Además, las pruebas inmunodiagnósticas disponibles no son útiles en el monitoreo pues detectan la presencia de anticuerpos contra $\operatorname{los} C$. cellulosae, los cuales se mantienen en circulación, aún después de la muerte de los quistes. Por ello, el objetivo del presente estudio fue demostrar la utilidad del ELISA captura de antígeno en el monitoreo de la persistencia de antígeno parasitario circulante en animales tratados contra cisticer-

\footnotetext{
${ }^{1}$ Práctica privada. E-mail: yenytinoco@speedy.com.pe

${ }^{2}$ Laboratorio de Medicina Veterinaria Preventiva, FMV-UNMSM

${ }^{3}$ Laboratorio de Microbiología y Parasitología Veterinaria, FMV-UNMSM
} 
cosis. Para este fin, se tomó un grupo de sueros que corresponden al seguimiento pre y post tratamiento de 6 cerdos naturalmente infectados con cisticercosis y tratados con oxfendazol. Se utilizó también el archivo de necropsias y las fichas de evaginación. Se encontró que el tiempo en el que los títulos de antígeno fueron inferiores al punto de corte ocurrió en el día 59 post tratamiento y que la media de supervivencia fue de 71.5 días (59.3-83.8). Los resultados parecen evidenciar que el ELISA-Ag permitiría el monitoreo cuantitativo del comportamiento y persistencia de los antígenos circulantes en animales naturalmente infectados y tratados con una droga cisticida. Sin embargo, se requiere monitorear mayor cantidad de cerdos para obtener resultados concluyentes.

Palabras clave: ELISA captura de antígeno, cisticercosis, monitoreo post tratamiento, antígenos parasitarios circulantes

\section{INTRODUCCIÓN}

La cisticercosis es una enfermedad zoonótica causada por el establecimiento de las formas larvarias de Taenia solium en el tejido de los hospederos intermediarios; principalmente el cerdo y accidentalmente el hombre. La cisticercosis está considerada a nivel mundial como un grave problema de salud pública, y los países en desarrollo como el Perú son comúnmente zonas endémicas de esta enfermedad (Reyes, 1991; Carpio, 2002).

El tratamiento de esta zoonosis es posible mediante el empleo de diferentes cisticidas como el oxfendazol que ha demostrado tener buenos resultados en el tratamiento de la cisticercosis muscular porcina (González et al., 1997, 1998). Sin embargo, es difícil el monitoreo y la evaluación del animal que está siendo tratado.

A la fecha, no existe un medio adecuado para el monitoreo de los individuos que han sido tratados contra cisticercosis, ya sean éstos humanos o cerdos (García et al., 1997). Las técnicas que han venido siendo empleadas para tal propósito son las de radioimagen (Tomografía Axial Computarizada y Resonancia Magnética), mientras que las pruebas diagnósticas de EITB y ELISA indirecto presentan limitaciones.
Las pruebas inmunodiagnósticas tales como el EITB y el ELISA indirecto detectan la presencia de anticuerpos contra Cysticercus cellulosae, pero estos anticuerpos continúan circulando en el suero luego de eliminado el quiste. En el caso de humanos se ha observado que los anticuerpos persisten hasta un año después de la curación de la enfermedad; situación que no permite utilizar estas pruebas en el monitoreo postratamiento de los individuos que han recibido terapia antiparasítica con un cisticida (Dorny et al., 2000; García et al., 1997).

Las técnicas de radioimagen como la Tomografía Axial Computarizada y la Resonancia Magnética son los únicos medios disponibles para hacer un seguimiento a pacientes luego del tratamiento (García et al., 1997; Carpio, 2002; Greene et al., 1999), pero estas técnicas son muy costosas, requieren aparatos muy sofisticados y son difíciles de obtener en las localidades donde la $T$. solium es endémica; añadiéndose a estos inconvenientes el hecho que sus resultados en forma independiente no son concluyentes (Figueroa, 1998).

Con la finalidad de poder diagnosticar la presencia de quistes viables en el organismo, se han venido desarrollando pruebas que detecten la presencia de antígeno del cisticerco por medio de anticuerpos policlonales y monoclonales en suero o líquido cefalorraquídeo de individuos afectados, 
ya sean humanos (Estrada et al., 1989; Correa et al., 1989; Chen et al., 1991; Cho et al., 1992; Wang et al., 1992; Wang et al., 1993; Lin et al., 1999) o cerdos (Harrison et al., 1989; Brandt et al., 1992; Draelants et al., 1995; Van Kerekhoven et al., 1998). De todas ellas, la prueba de ELISA de captura de antígeno que utiliza anticuerpos monoclonales demostró ser la más adecuada en la detección de los antígenos de secreción-excreción de Cysticercus cellulosae (Harrison et al., 1989; Brandt et al., 1992). Esto daría la oportunidad de un diagnóstico confiable de la enfermedad, al mismo tiempo que determinaría la situación real del individuo y con ello su uso en el monitoreo y seguimiento de animales tratados. Se añade a estas ventajas el hecho de ser una prueba reproducible y de menor costo que las técnicas de radioimagen.

El presente trabajo tuvo por objetivo demostrar la utilidad del ELISA captura de antígeno en el monitoreo de cerdos naturalmente infectados y tratados con un antiparasitario (oxfendazol).

\section{Materiales y Métodos}

\section{Lugar de estudio}

El estudio se llevó a cabo en el Laboratorio de Parasitología de la Facultad de Medicina Veterinaria de la Universidad Nacional Mayor de San Marcos, Lima.

\section{Material biológico}

Las muestras de suero pertenecen al Banco de Suero del Cysticercosis Working Group of Peru. La historia de los sueros indica que corresponden al seguimiento pre y post tratamiento de seis cerdos, escogidos al azar y sin parentesco alguno, de aproximadamente 8 meses de edad y $45 \mathrm{~kg}$ de peso vivo. Estos cerdos fueron naturalmente infectados con huevos de $T$. solium y adquiridos en
Huancayo (lugar donde la cisticercosis es endémica en la crianza extensiva de cerdos). El diagnóstico de positividad de los cerdos previo al tratamiento se realizó mediante la prueba de la lengua y del EITB. El tratamiento se hizo con dosificaciones orales con Oxfendazol, Synanthic ${ }^{\circledR}$ al $2.265 \%$ (30 mg/kg de peso vivo). Dos cerdos fueron tratados con una sola dosis y cuatro cerdos con dos dosis con una semana de intervalo.

Se tomaron 24 muestras por animal (5 muestras antes del primer tratamiento, una muestra el mismo día del tratamiento y 18 muestras posteriores al primer tratamiento) con un intervalo de 4 días aproximadamente. Se realizó la necropsia de los cerdos al cabo de 12 semanas del tratamiento y se tomaron muestras de los cisticercos para realizar pruebas de viabilidad (evaginación de los cisticercos en bilis bovina a $37{ }^{\circ} \mathrm{C}$ durante dos horas).

Se utilizó el ELISA-Ag descrito por Van Kerekhoven et al. (1998). Los anticuerpos monoclonales utilizados en la prueba B158C $_{11} \mathrm{~A}_{10}$ y B60H $\mathrm{H}_{8} \mathrm{~A}_{4}$ Biotinilado) fueron proporcionados por el Dr. Jeff Brandt (Prince Leopold Institute of Tropical Medicine, Bélgica).

\section{Análisis de datos}

El punto de corte estuvo representado por el límite inferior del intervalo de confianza de la media de los títulos de antígenos de los animales infectados previo al tratamiento (Daniel, 1995). La media fue de 1.866, la desviación estándar de 0.76117 y el punto de corte fue de 0.3436. Los datos fueron analizados mediante el análisis de supervivencia de Kaplan Meier.

\section{Resultados}

La prueba diagnóstica de ELISA de captura de antígeno permitió monitorear la 


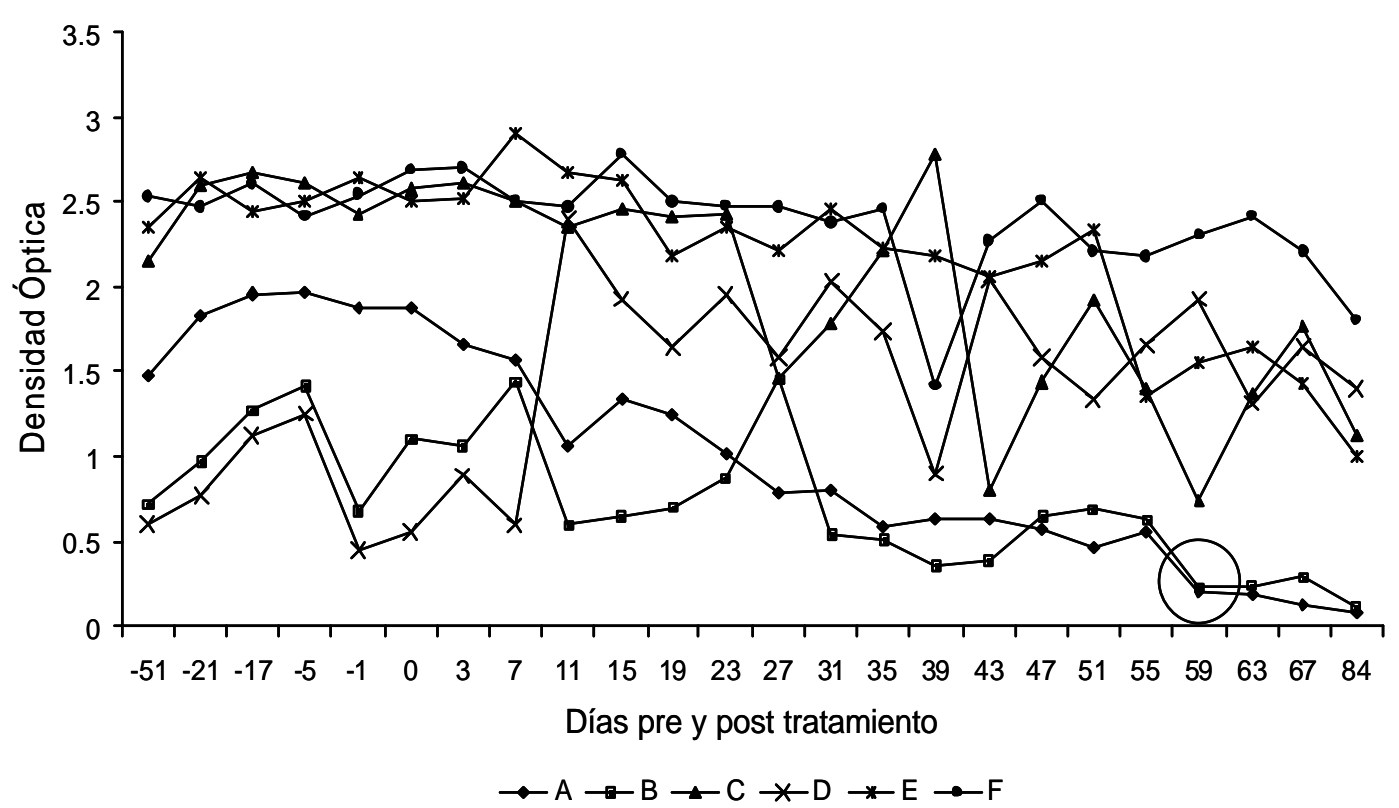

Figura 1. Monitoreo de antígeno parasitario en suero de cerdos con cisticercosis, naturalmente infectados y tratados con oxfendazol

persistencia de antígeno parasitario circulante de Cysticercus cellulosae en cerdos naturalmente infectados y tratados con oxfendazol (Fig. 1). Los títulos de los antígenos circulantes en suero comenzaron a ser menores al punto de corte el día 59 posterior al primer tratamiento. Los animales con 3 tratamientos $(n=4)$ tuvieron una media de supervivencia de 71.5 días (IC: 59.3 - 83.8), tiempo medio en el que la cantidad de antígenos parasitarios en suero, expresados en densidades ópticas, descendieron por debajo del punto de corte. Los dos animales con un sólo tratamiento no pudieron ser evaluados, pues en ninguno de ellos se produjo el evento (hasta el final del experimento las densidades ópticas fueron mayores al punto de corte).

Los resultados hallados en las fichas de necropsia muestran que los animales que presentan escasa carga parasitaria (número de quistes por gramo de tejido) tienen a su vez pequeñas cantidades de antígeno parasitario circulante al final del monitoreo; a diferencia de los animales que presentan alta carga pa- rasitaria y tienen a la vez, altas cantidades de antígeno circulante al final del estudio (Cuadro 1).

Los resultados de la prueba de evaginación muestran que los únicos cisticercos que evaginaron fueron aquellos que se encontraron en el cerebro de cuatro cerdos. Los porcentajes de evaginación encontrados varían entre 31 y $100 \%$. Los demás quistes, aunque aparentemente normales, no fueron viables cuando fueron sometidos a la prueba de evaginación (Cuadro 2).

\section{Discusión}

Los resultados del presente estudio demostrarían la utilidad de la prueba ELISA$\mathrm{Ag}$ en el monitoreo de antígenos circulantes de secreción-excreción de Cysticercus cellulosae en animales naturalmente infectados en el periodo postratamiento de la enfermedad. Esta prueba permitió evaluar el comportamiento de los antígenos de secre- 
Cuadro 1. Quistes ${ }^{1}$ por gramo de muestra de tejido $\left(\mathrm{Q} / \mathrm{P}^{2}\right)$ colectada en la necropsia ${ }^{3}$ de cerdos infectados naturalmente con Cysticercus cellulosae y tratados con oxfendazol

\begin{tabular}{|c|c|c|c|c|c|c|c|c|c|c|c|c|}
\hline & \multicolumn{3}{|c|}{ Cerebro } & \multicolumn{3}{|c|}{ Lengua } & \multicolumn{3}{|c|}{ Corazón } & \multicolumn{3}{|c|}{ Músculo } \\
\hline & Peso & Quistes & $\mathrm{Q} / \mathrm{P}$ & Peso & Quistes & $\mathrm{Q} / \mathrm{P}$ & Peso & Quistes & $\mathrm{Q} / \mathrm{P}$ & Peso & Quistes & $\mathrm{Q} / \mathrm{P}$ \\
\hline \multicolumn{13}{|c|}{ Curados } \\
\hline A & 39.5 & 0 & 0 & 13.9 & 0 & 0 & 32.2 & 0 & 0 & 37.0 & 1 & 0.03 \\
\hline $\mathrm{B}$ & 13.9 & 0 & 0 & 24.7 & 1 & 0.04 & 18.4 & 1 & 0.05 & 26.3 & 3 & 0.11 \\
\hline \multicolumn{13}{|c|}{ No curados } \\
\hline $\mathrm{C}$ & 9.5 & 20 & 2.11 & 8.0 & 12 & 1.50 & 12.5 & 3 & 0.24 & 18.7 & 14 & 0.75 \\
\hline $\mathrm{D}$ & 11.4 & 2 & 0.18 & 14.9 & 1 & 0.07 & 8.7 & 3 & 0.34 & 26.7 & 1 & 0.04 \\
\hline $\mathrm{E}$ & 8.5 & 0 & 0 & 12 & 0 & 0 & 14.5 & 2 & 0.10 & 38.5 & 11 & 0.30 \\
\hline $\mathrm{F}$ & 10.5 & 3 & 0.30 & 21.7 & 13 & 0.60 & 13.0 & 0 & 0 & 31.0 & 82 & 2.60 \\
\hline
\end{tabular}

${ }^{1}$ Incluye quistes degenerados y de apariencia normal

2 Relación de quistes por gramo de muestra de tejido (refleja la carga parasitaria del animal)

${ }^{3} \mathrm{~A}$ las 12 semanas del tratamiento con oxfendazol

Cuadro 2. Viabilidad de cisticercos ${ }^{1}$ aparentemente normales, mediante la prueba de evaginación, en tejidos de cerdos infectados naturalmente con Cysticercus cellulosae y tratados con oxfendazol

\begin{tabular}{lllllllllllll}
\hline & \multicolumn{3}{c}{ Cerebro } & \multicolumn{2}{c}{ Lengua } & \multicolumn{3}{c}{ Corazón } & \multicolumn{3}{c}{ Músculo } \\
\cline { 2 - 10 } & Evag $^{2}$ & Viab $^{3}$ & $\%^{4}$ & Evag & Viab & $\%$ & Evag & Viab & $\%$ & Evag & Viab & $\%$ \\
\hline
\end{tabular}

Curados

$\begin{array}{ccccccccccccc}\text { A } & 1 & 1 & 100 & 1 & 0 & 0 & 0 & 0 & - & 6 & 0 & 0 \\ \text { B } & 1 & 0 & 0 & 9 & 0 & 0 & 3 & 0 & 0 & 2 & 0 & 0\end{array}$

No curados

\begin{tabular}{ccccccccccccc}
$\mathrm{C}$ & 35 & 11 & 31 & 0 & 0 & - & 3 & 0 & 0 & 0 & 0 & - \\
$\mathrm{D}$ & 3 & 2 & 67 & 18 & 0 & 0 & 18 & 0 & 0 & 5 & 0 & 0 \\
$\mathrm{E}$ & 2 & 1 & 50 & 12 & 0 & 0 & 33 & 0 & 0 & 40 & 0 & 0 \\
$\mathrm{~F}$ & 3 & 0 & 0 & 40 & 0 & 0 & 5 & 0 & 0 & 40 & 0 & 0 \\
\hline
\end{tabular}

${ }^{1}$ Cisticercos aparentemente viables encontrados en la evaluación completa del animal a las 12 semanas del tratamiento con oxfendazol

2 Cisticercos evaluados con la prueba de evaginación

${ }^{3}$ Cisticercos que evaginaron en la prueba

${ }^{4}$ Porcentaje de viabilidad 
ción-excreción como consecuencia de la destrucción del quiste por efecto del tratamiento antiparasitario aplicado.

El adecuado monitoreo de los antígenos circulantes haría del ELISA-Ag una adecuada herramienta en el seguimiento de la evolución de la infección en individuos con tratamiento contra cisticercosis por Taenia solium; más aún si se compara con la Tomografía Axial Computarizada y la Resonancia Magnética como medios de seguimiento y monitoreo de individuos tratados, dado que es una técnica económica, reproducible y de fácil acceso en lugares donde la cisticercosis es endémica (García et al., 2000; Benítez et al., 2001a, b; Brandt et al., 2001).

La media de supervivencia encontrada fue de 71.5 días (IC: 59.3 - 83.8) postratamiento, evento que está relacionado con la información hallada a la necropsia de cerdos tratados con el oxfendazol, donde se observó la degeneración de los quistes a partir de la semana 8 del tratamiento (González et al., 1998). Esto explicaría la reducción de antígeno parasitario circulante en el suero de dos cerdos a partir del día 59 postratamiento (Fig. 1).

Los dos cerdos que alcanzaron densidades ópticas por debajo del punto de corte a partir del día 59 postratamiento podrían considerse como curados. Los datos de las necropsias confirman estos resultados, toda vez que presentaron escasa presencia de quistes totales (quistes aparentemente normales y degenerados) por gramo de muestra en los diferentes órganos evaluados y ningún quiste viable con la prueba de evaginación en los músculos evaluados (Cuadro 2); sin embargo, se debe indicar que uno de los cerdos considerado como curado presentó un cisticerco viable en el cerebro por lo que la baja cantidad de antígeno circulante en suero de este animal puede deberse a la variación de la actividad metabólica del cisticerco o a las diferencias de permeabilidad de la cápsula de tejido conectivo alrededor del quiste (Nguekam et al., 2003).
Los cuatro animales que mantuvieron valores de densidad óptica mayor al punto de corte hasta el final del monitoreo fueron considerados como no curados. Los títulos de antígeno elevados en estos animales pueden estar relacionados al alto grado de infección o carga parasitaria (Cuadro 1), además del mayor número de quistes aparentemente normales evaluados mediante la prueba de evaginación en comparación con los animales considerados curados (Cuadro 2). Estos quistes aparentemente normales puestos a evaginar y que no fueron viables a la prueba de evaginación, pueden haber muerto recientemente y sus antígenos parasitarios haberse mantenido en circulación al momento de la toma de la última muestra.

La presencia de quistes viables en el cerebro de tres de los cerdos no curados (Cuadro 2), concuerda con los reportes realizados acerca del tratamiento con oxfendazol que es efectivo contra la cisticercosis muscular porcina, pero es deficiente en la neurocisticercosis (González et al., 1997; Falcón, 1995).

La prueba de ELISA-Ag ha sido utilizada anteriormente en el monitoreo de animales infectados en forma experimental (Brandt et al., 1992). Allí se evaluó el comportamiento de los antígenos parasitarios en suero de bovinos infectados con diferentes dosis de huevos de Taenia saginata encontrando que los antígenos se encontraban presentes en forma ascendente en aquellos animales que lograron infectarse. Posteriormente, Nguekam et al. (2003) realizaron un trabajo similar en cerdos, encontrando que en cerdos negativos a la presencia de antígeno circulante al inicio de la prueba, éste fue apareciendo en aquellos animales que consiguieron infectarse.

En humanos, García et al. (2000) encontraron que el antígeno parasitario circulante en sueros de pacientes humanos curados de neurocisticercosis (diagnosticados por Tomografía Axial Computarizada) disminuyó rápidamente en el transcurso de tres meses 
luego del tratamiento a diferencia de los pacientes no curados. Por el contrario, en otro trabajo del mismo autor (García et al., 1997) se hizo un seguimiento serológico utilizando el EITB a humanos tratados contra cisticercosis, encontrando seropositividad hasta un año después de ser considerados como "curados" por Tomografía Axial Computarizada, confirmando que los anticuerpos se mantienen en circulación tiempo después de la muerte del cisticerco (Correa et al., 1989; García et al., 1997; Dorny et al., 2000).

La diferencia entre el ELISA-Ag utilizado en el presente estudio y el utilizado por García et al. (2000) es el isotipo de anticuerpo monoclonal empleado. Los anticuerpos monoclonales del isotipo Ig $\mathrm{G}$ utilizados en el presente estudio poseen mayor afinidad por los antígenos de secreción-excreción, y además son más estables (Van Kerekhoven et al., 1998), lo que brinda una ventaja a la prueba en términos diagnósticos y de monitoreo comparada a la utilizada por García et al. (2000) que hacen uso de anticuerpos monoclonales del isotipo Ig $\mathrm{M}$.

\section{Conclusiones}

Se tiene evidencia que el ELISA-Ag podría monitorear cuantitativamente los niveles de antígeno parasitario circulante en suero de cerdos naturalmente infectados y tratados con un cisticida. Sin embargo, se hace necesario realizar monitoreos adicionales en mayor cantidad de animales que respondan a los cisticidas a fin de validar la utilidad del método en cerdos.

\section{Agradecimientos}

Nuestros sinceros agradecimientos a los doctores Néstor Falcón y Francisco Suárez por las revisiones realizadas al presente trabajo, y al Cysticercosis Working Group of Peru por el apoyo prestado.

\section{Literatura Citada}

1. Benítez, O.; M. Barrionuevo; J. Ron; M. Chávez; R. Rodríguez; M. Cruz; J. Brandt; S. Geerts; E. Van Marck; A. Ito; S. Lima. 2001a. Inmunodiagnóstico de cisticercosis por ELISA-Ag (ICCE): aplicación en el hombre y en los cerdos. En: Cuadernos del ISIP. Rev. Fac. Med. Vet. y Zoot., Univ. Central del Ecuador. Benítez, W. (ed). Vol II. p 22-28. Memorias del International Workshop El Complejo Teniasis - Cisticercosis.

2. Benítez, O.; J. Ron; M. Barrionuevo; R. Rodríguez; M. Chávez; P. Proaño; J. Brandt; S. Geerts; E. Van Marck; A. Ito; S. Lima. 2001b. Tratamiento de la cisticercosis porcina, utilizando dos dosis de oxfendazol. Resultados preliminares. En: Cuadernos del ISIP. Rev. Fac. Med. Vet. y Zoot., Univ. Central del Ecuador. Benítez, W. (ed). Vol II. p 221231. Memorias del International Workshop El Complejo Teniasis - Cisticercosis.

3. Brandt, J.; S. Geerts; R. De Deken; V. Kumar; F. Ceulemns; L. Brus; N. Falla. 1992. A monoclonal antibodybased ELISA for the detection of circulating excretory-secretory antigens in Taenia saginata cysticercosis. Internat. J. Parasitol. 22: 471-477.

4. Brandt, J.; S. Geerts; P. Dorny. 2001. Desarrollo de la prueba ELISA para la detección de antígenos en circulación de la cisticercosis en base a dos anticuerpos monoclonales. En: Cuadernos del ISIP. Rev. Fac. Med. Vet. y Zoot., Univ. Central del Ecuador. Benítez, W. (ed). Vol II. p 19-21. Memorias del International Worksop El Complejo Teniasis - Cisticercosis.

5. Carpio, A. 2002. Neurocysticercosis: an update. Lancet Infect. Dis. 2: 751-762.

6. Chen, J.; X. Zhang; W. Tan; M. Liu; G. Liu; Y. Hu. 1991. Determination of circulating antigen in cysticercosis patients using McAb-based ELISA. 
Zhongguo Ji Sheng Chong Xue Ji Sheng Chong Bing Za Zhi 9: 122-125. [En chino con resumen en inglés].

7. Cho, S.; Y. Kong; S. Kim; S. Kang. 1992. Measurement of $150 \mathrm{kDa}$ protein of Taenia solium metacestodes by antibody-sandwich ELISA in cerebrospinal fluid of neurocysticercosis patients. Kisaengchunghak Chapchi 30: 299-307. [En chino con resumen en inglés].

8. Correa, D.; M. Sandoval; L. Harrison; M. Parkhouse; A. Plancarte; A. Meza-Lucas; A. Flisser. 1989. Human neurocysticercosis: Comparisons of enzyme immunoassay capture techniques based on monoclonal and polyclonal antibodies for the detection of parasite products in cerebrospinal fluid. Trans. R. Soc. Trop. Med. Hyg. 83: 814-816.

9. Daniel, W.D. 1995. Bioestadística. Base para el análisis de las ciencias de la salud. p 130-140. UTA. México.

10. Dorny, P.; F. Vercammen; J. Brandt; W. Vansteenkiste; D. Berkvens; S. Geerts. 2000. Sero-epidemiological study of Taenia saginata cysticercosis in Belgian cattle. Vet. Parasitol. 88: 43-49.

11. Draelants, E.; E. Hofkens; E. Harding; J. Brandt; S. Geerts. 1995. Development of a dot-enzyme immunoassay for the detection of circulating antigen in cattle infected with Taenia saginata cysticerci. Res. Vet. Sci. 58: 99-100.

12. Estrada, J.; J.A. Estrada; R. Kuhn. 1989. Identification of Taenia solium antigens in cerebroespinal fluid and larval antigens from patients with neurocysticercosis. Am. J. Trop. Med. Hyg. 41: 50-55.

13. Falcón, N. 1995. Uso del oxfendazole en el tratamiento de cisticercosis porcina. Tesis de Bachiller. Facultad de Medicina Veterinaria, Univ. Nacional Mayor de San Marcos. Lima. 51 p.

14. Figueroa, J. 1998. Tomografía Axial Computarizada y Resonancia Magnética Nuclear en el diagnóstico de neurocisticercosis. Comparación de sensibilidades. Tesis de Bachiller. Facultad de Medicina Humana, Univ. Peruana Cayetano Heredia. Lima. 80 p.

15. García, H.; R. Gilman; M. Catacora; M. Verástegui; A. González; V. Tsang; Cysticercosis Working Group of Peru. 1997. Serologic evolution of neurocysticercosis patients after antiparasitic therapy. JID. 175: 486-489.

16. García, H.; R. Parkhouse; R. Gilman; T. Montenegro; T. Bernal; S. Martínez; A. González; V. Tsang; L. Harrison. 2000. Serum antigen detection in the diagnosis, treatment, and follow-up of neurocysticercosis patients. Trans. R. Soc. Trop. Med. Hyg. 94: 673676.

17. González, A.; N. Falcón; C. Gavidia; H. García; V. Tsang; T. Bernal; M. Romero; R. Gilman. 1997. Treatment of swine cysticercosis with oxfendazole: a dose response trial. Vet. Rec. 141: 420-422.

18. González, A.; N. Falcón; C. Gavidia; H. García; V. Tsang; T. Bernal; et al. 1998. Time response curve of oxfendazole in the treatment of swine cysticercosis. Am. J. Trop. Med. Hyg. 59: 832-836.

19. González, A.; C. Gavidia; N. Falcón; C. Evans; T. Bernal; T. López; H. García; R. Gilman. 1999. Porcine cisticercosis: Epidemiology, diagnosis and treatment. En: Taenia solium Taeniasis/Cysticercosis. García, H.; S. Martínez (eds). p97-119. Ed. Universo. Lima.

20. Greene, R.; P. Wilkins; V. Tsang. 1999. Diagnostic glycoproteins of Taenia solium cysts share homologus 14- and 18-kDa subunits. Molec. Biochem. Parasitol. 99: 257-261.

21. Harrison, L.; G. Joshua; S. Wright; R. Parkhouse. 1989. Specific detection of circulating surface/secreted glycoproteins of variable cysticerci in Taenia saginata cysticercosis. Parasite Immunology 11: 351-370. 
22. Lin, X.; G. Li; H. Huo; F. Xu; Q. Li; Z. Zhao. 1999. Detection of circulating antigen in serum and cerobroespinal fluid for diagnosis of cerebral cysticercosis. Zhongguo Ji Sheng Chong Xue Ji Sheng Chong Bing Za Zhi 17: 146-148. [En chino con resumen en inglés].

23. Nguekam, A.; P. Zoli; L. Vondou; S. Pouedet; E. Assana; P. Dorny; J. Brandt; B. Losson; S. Geerts. 2003. Kinetics of circulating antigens in pigs experimentally infected with Taenia solium eggs. Vet. Parasitol. 111: 323332.

24. Reyes, H. 1991. Cisticercosis. En: Parasitología Clínica. Atias A. (ed). p 351-359. Publicaciones Técnicas Mediterráneo. Chile.
25. Van Kerekhoven, I.; M. Vanteenkiste; M. Claes; S. Geerts; J. Brandt. 1998. Improved detection of circulating antigen in cattle infected with Taenia saginata metacestodes. Vet. Parasitol. 76: 269274.

26. Wang, C.; H. Zhang; L. Ge. 1992. A Mab-based ELISA for detecting circulating antigen in CSF of patients with neurocysticercosis. Hibrinoma 11: 825827.

27. Wang, C.; Q. Li; H. Zhang; J. Su; J. Zhang; H. Li; F. Jia; L. Ge. 1993. Detection of CAg in CSF of 231 cerebral cysticercosis patients. Zhongguo Ji Sheng Chong Xue Ji Sheng Chong Bing Za Zhi 11: 276-278. [En chino con resumen en inglés]. 